(1)

CrossMark

\title{
Pulmonary embolism in patients with COVID-19 pneumonia
}

\author{
To the Editor:
}

Acute respiratory distress syndrome development in patients with coronavirus disease 2019 (COVID-19) pneumonia is associated with a high mortality rate and is the main cause of death in patients with severe acute respiratory syndrome coronavirus 2 (SARS-CoV-2) infection [1]. Myocardial injury has also been reported to be significantly associated with fatal outcome, with a $37 \%$ mortality rate in patients without prior cardiovascular disease but elevated troponin levels [2]. A D-dimer level of $>1 \mu \mathrm{g} \cdot \mathrm{mL}^{-1}$ has been clearly identified as a risk factor for poor outcome in SARS-Cov-2 infection [3], with recent reports highlighting a high incidence of thrombotic events in intensive care unit (ICU) patients [4]. A normal D-dimer level allows the safe exclusion of pulmonary embolism (PE) in outpatients with a low or intermediate clinical probability of $\mathrm{PE}$, but there is no recommendation to use D-dimer as a positive marker of thrombosis because of lack of specificity. An advice paper from the European Society of Radiology (ESR) and the European Society of Thoracic Imaging (ESTI) suggested that contrast-enhanced computed tomography (CT) should be performed to rule out PE if supplementary oxygen is needed in COVID-19 pneumonia patients with limited disease extension [5]. The European Society of Cardiology (ESC) also recommends that CT pulmonary angiography (CTPA) should be performed when unenhanced CT findings cannot explain the severity of respiratory failure [6]. Indeed, it is not recommended that PE diagnosis should be based on pulmonary ventilation-perfusion imaging [7]. The cumulative incidence of venous thromboembolism (VTE) on the wards is reported to be lower than in the ICU (10\% versus $48 \%$ at 14 days, respectively) [8], where investigation for PE is not always feasible due to critical illness and prone position [9].

We conducted a retrospective study evaluating all CTPAs performed between 1 March and 16 April 2020, in patients with COVID-19 pneumonia at two hospitals of Université de Paris (Cochin and Pompidou hospitals, Paris, France). The study was approved by the ethics committee of Cochin Hospital (CLEP Decision no. AAA-2020-08009). The reason patients had been referred for CTPA was obtained from the electronic medical records, as well as the number of days of hospitalisation at the time of CT examination, the categorisation as ICU or ward patient, and the need for mechanical ventilation. Information regarding D-dimer level within $24 \mathrm{~h}$ of the CT examination was also obtained, together with the modalities of prophylactic anticoagulation in hospitalised patients. CT acquisitions were performed with multidetector CT units (SOMATOM Definition Edge or SOMATOM Definition AS; both Siemens Healthineers, Erlangen, Germany) using standard CTPA protocols. Images were analysed by two experienced radiologists who evaluated COVID-19 disease extent, the presence or absence of intraluminal filling defects in the proximal or distal (segmental or subsegmental) pulmonary arteries and signs of acute right ventricular dysfunction.

During the study period, a total of 137 patients with confirmed SARS-Cov-2 infection and COVID-19 pneumonia underwent contrast-enhanced CT examination. All but two CT examinations with major respiratory motion artifacts were of diagnostic quality, as assessed by the two expert readers. Thus, 135 patients (94 men, 70\%) with a median (interquartile range (IQR)) age of 64 years (54-76 years) were included. $63(47 \%)$ of them were outpatients seen at the emergency department. They were referred for CTPA at initial presentation because of hesitation between COVID-19 pneumonia and PE, after clinical probability assessment and D-dimer dosage, applying the same cut-off as for non-COVID patients. The remaining $72(53 \%)$ patients were already hospitalised for a median of 5 days (IQR 4-8 days) and had

@ERSpublications

This study reports an overall $24 \%$ (95\% CI $17-32 \%)$ cumulative incidence of pulmonary embolism in patients with COVID-19 pneumonia, $50 \%(30-70 \%)$ in ICU and $18 \%(12-27 \%)$ in other patients https://bit.ly/35s7hjm

Cite this article as: Bompard F, Monnier H, Saab I, et al. Pulmonary embolism in patients with COVID-19 pneumonia. Eur Respir J 2020; 56: 2001365 [https://doi.org/10.1183/13993003.01365-2020]. 
presented clinical respiratory deterioration, with an increased need for oxygen. 24 patients were in ICU under mechanical ventilation.

There was no disagreement between the two expert readers at initial and expert reading regarding the presence of PE. A total of $32 \mathrm{PEs}$ were identified, resulting in a $24 \%$ (95\% CI 17-32\%) overall cumulative incidence, $50 \%$ (95\% CI 30-70\%) in ICU patients and 18\% (95\% CI 12-27\%) in other patients. 15 PEs were diagnosed in outpatients at initial presentation, whereas the remaining 17 were diagnosed in patients who had presented clinical deterioration during hospitalisation. All inpatients received prophylactic anticoagulation (enoxaparin $40 \mathrm{mg}$ once daily in ward patients, or twice daily in obese and ICU patients according to local practice). There were 10 proximal PEs and 22 peripheral PEs, 18 of which were involved segmental pulmonary arteries and four were involved in multiple subsegmental pulmonary arteries. On CTPA there were signs of acute right ventricular dysfunction (right-to-left ventricular diameter ratio $>1$ ) in six patients, four with proximal PE including one saddle PE, and two others with segmental PE, of which one patient had moderate and the other had severe disease extent on CT.

Comparison between the PE-positive and PE-negative groups is summarised in the table 1. No statistical difference between groups was noted regarding age or sex.

Interestingly, disease extent on CTPA was not significantly different between patients with or without PE. Conversely, among the 53 patients referred as a result of clinical respiratory deterioration who had a previous CT, an increase in disease extent was more frequently observed in the PE-negative group. Patients with PE were more frequently hospitalised in ICU and more frequently under mechanical ventilation, with a longer median (IQR) hospitalisation duration $(15$ (9-17) versus 8 (4-12) days; $\mathrm{p}=0.04)$. There were four deaths in the PE-positive group (four out of 32,13\%) and 12 in the PE-negative group (12 out of 135, $12 \%)$, within a median (IQR) follow-up of 26 (22-29) and 27 (20-32) days, respectively.

D-dimer level was significantly higher in the PE group; meaning the D-dimer increase is not only a marker of pneumonia severity but is also associated with a higher risk of PE. Our data do not allow for the definition of an accurate threshold for PE risk in this specific population. However, the high cumulative incidence of $\mathrm{PE}$ we observed suggests contrast medium should be more frequently used for the evaluation of COVID-19 patients on CT.

Some authors suggested using low molecular weight heparin anticoagulant therapy in patients with severe pneumonia who have excessive activation of coagulation, defined as D-Dimer values four times higher than the normal upper limit [10]. TANG et al. [11] reported lower 28-day mortality in patients with D-dimer more than six fold of upper limit of normal who received heparin at a prophylactic dose, as compared with those who did not.

TABLE 1 Comparison of pulmonary embolism (PE)-positive and PE-negative groups

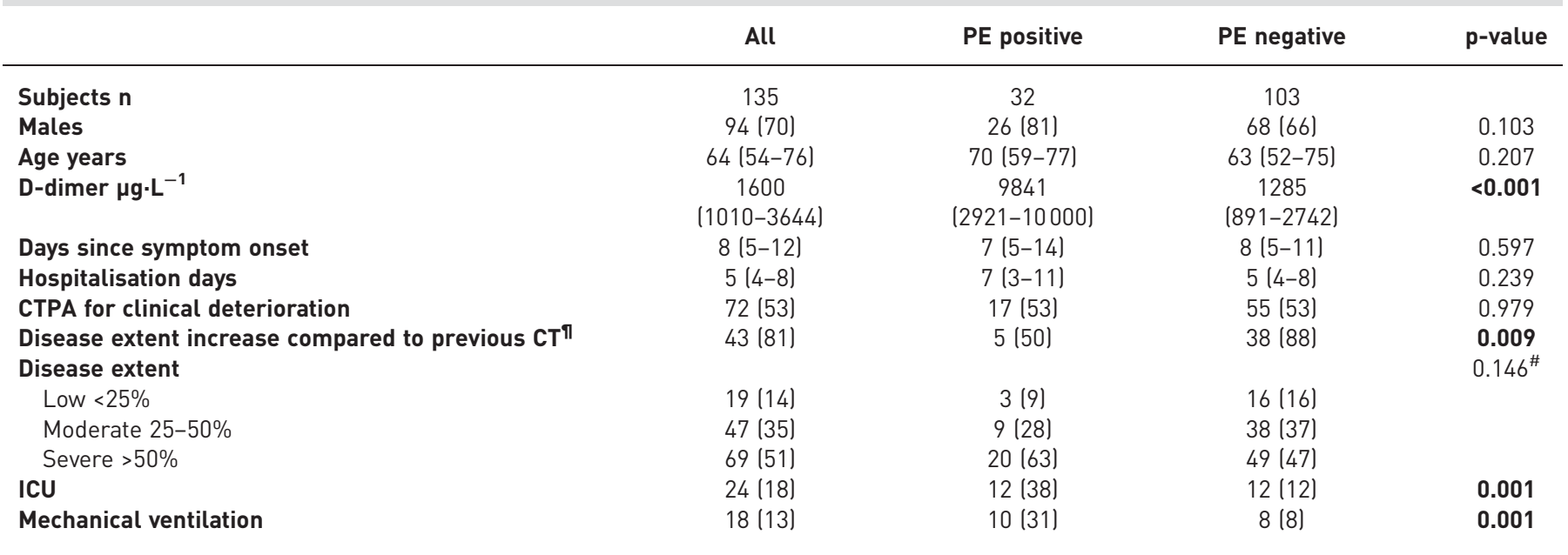

Categorical variables are presented as $\mathrm{n}(\%)$ and comparisons between the two groups were made using the Chi-squared test. Continuous variables are presented as median (interquartile range) and comparisons between the two groups were made using the Mann-Whitney U-test. Bold indicates statistical significance. CTPA: computed tomography pulmonary angiography; CT: computed tomography; ICU: intensive care unit. ": Cochran-Armitage trend test; ${ }^{\#}$ : only 53 of the 72 inpatients had a previous CT. 
In our study, prophylactic anticoagulation did not avoid the occurrence of PE in hospitalised patients, highlighting the need to adapt thrombosis prophylaxis in patients with SARS-Cov-2 infection.

$\mathrm{PE}$ and deep venous thrombosis (DVT) have been reported to occur in other viral pneumonias, but not as frequently as in COVID-19 patients. Of 119 individuals admitted to the hospital with H1N1 influenza A virus infection, seven (5.9\%) experienced thrombotic vascular events, including one PE and three DVT [12].

Our study presents several limitations. As contrast administration was not systematically used, our study does not allow assessment of the incidence of PE in all COVID-19 patients. Although all patients referred because of clinical deterioration underwent a contrast-enhanced CT, the reason PE was suspected as a concurrent diagnosis of COVID-19 pneumonia at initial presentation cannot be precisely assessed, as a result of the retrospective design of our study. For the same reason, an in-depth analysis of comorbidities and particularly the history of VTE was not possible. We were not able to evaluate the presence of DVT in our study population as none of the patients underwent compression ultrasound. Lastly, our study does not allow us to determine which D-dimer cut-off value triggers contrast administration, when evaluating COVID-19 patients on CT. This remains an open question to be solved by a prospective evaluation.

In conclusion, we observed that almost a quarter of the COVID-19 pneumonia patients evaluated after contrast administration had acute PE on CT. This suggests contrast-enhanced CT should be more widely used when assessing patients with COVID-19 pneumonia, particularly in those with marked elevation of D-dimer.

Florian Bompard ${ }^{1}$, Hippolyte Monnier ${ }^{2}$, Ines Saab ${ }^{1,3}$, Mickael Tordjman $^{1}$, Hendy Abdoul ${ }^{4}$, Laure Fournier ${ }^{2,3}$, Olivier Sanchez $^{3,5}$, Christine Lorut ${ }^{6}$, Guillaume Chassagnon ${ }^{1,3}$ and Marie-Pierre Revel $\circledast^{1,3}$

${ }^{1}$ Dept of Radiology, Cochin Hospital, AP-HP Centre, Paris, France. ${ }^{2}$ Dept of Radiology, Hôpital Européen GeorgesPompidou, AP-HP Centre, Paris, France. ${ }^{3}$ Université de Paris, Descartes-Paris 5, Paris, France. ${ }^{4}$ Unité de Recherche Clinique Centre d'Investigation Clinique, Paris Descartes Necker/Cochin, Hôpital Tarnier, Paris, France. ${ }^{5}$ Dept of Pulmonology and Intensive Care, Hôpital Européen Georges-Pompidou, AP-HP Centre, Paris, France. ${ }^{6}$ Dept of Pulmonology, Cochin Hospital, AP-HP Centre, Paris, France.

Correspondence: Marie-pierre Revel, Dept of Radiology, Cochin Hospital, 27 rue du Faubourg Saint Jacques, 75014 Paris, France. E-mail: marie-pierre.revel@aphp.fr

Received: 23 April 2020 | Accepted after revision: 29 April 2020

This article has editorial commentaries: https://doi.org/10.1183/13993003.01608-2020 and https://doi.org/10.1183/ 13993003.01634-2020

Conflict of interest: F. Bompard has nothing to disclose. H. Monnier has nothing to disclose. I. Saab has nothing to disclose. M. Tordjman has nothing to disclose. H. Abdoul has nothing to disclose. L. Fournier reports personal fees for lectures from General Electric, Janssen and Novartis, non-financial support from Guerbet, outside the submitted work. O. Sanchez reports personal fees and non-financial support from BTG, grants and personal fees from Boston Scientific, grants, personal fees and non-financial support from Bayer and BMS, personal fees from Sanofi Aventis, grants from Daiichi Sankyo, during the conduct of the study; grants, personal fees and non-financial support from MSD, grants from Boehringer Ingelheim, outside the submitted work. C. Lorut reports non-financial support from Covidien AG, Novatech SA and Proxilio, outside the submitted work. G. Chassagnon has nothing to disclose. M-P. Revel reports personal fees for lectures from MSD France, travel support from Guerbet, outside the submitted work.

\section{References}

1 Wu C, Chen X, Cai Y, et al. Risk factors associated with acute respiratory distress syndrome and death in patients with coronavirus disease 2019 pneumonia in Wuhan, China. JAMA Intern Med 2020; 180: 1-11.

2 Guo T, Fan Y, Chen M, et al. Cardiovascular implications of fatal outcomes of patients with coronavirus disease 2019 (COVID-19). JAMA Cardiol 2020; 5: 1-8.

3 Tang N, Li D, Wang X, et al. Abnormal coagulation parameters are associated with poor prognosis in patients with novel coronavirus pneumonia. J Thromb Haemost 2020; 18: 844-847.

4 Klok FA, Kruip MJHA, van der Meer NJM, et al. Incidence of thrombotic complications in critically ill ICU patients with COVID-19. Thromb Res 2020; 191: 145-147.

5 Revel M-P, Parkar AP, Prosch H, et al. COVID-19 patients and the radiology department - advice from the European Society of Radiology (ESR) and the European Society of Thoracic Imaging (ESTI). Eur Radiol; in press [https://doi.org/10.1007/s00330-020-06865-y].

6 ESC Guidance for the Diagnosis and Management of CV Disease during the COVID-19 Pandemic. www.escardio. org/Education/COVID-19-and-Cardiology/ESC-COVID-19-Guidance Date last updated: 21 April 2020. Date last accessed: 23 April 2020.

7 Zhai Z, Li C, Chen Y, et al. Prevention and treatment of venous thromboembolism associated with coronavirus disease 2019 infection: a consensus statement before guidelines. Thromb Haemost 2020; 120: 937-948.

8 Middeldorp S, Coppens M, van Haaps TF, et al. Incidence of venous thromboembolism in hospitalized patients with COVID-19. Preprints 2020; preprint [https://10.20944/preprints202004.0345.v1].

9 Bikdeli B, Madhavan MV, Jimenez D, et al. COVID-19 and thrombotic or thromboembolic disease: implications for prevention, antithrombotic therapy, and follow-up. J Am Coll Cardiol 2020; 75: 2950-2973. 
10 Lin L, Lu L, Cao W, et al. Hypothesis for potential pathogenesis of SARS-CoV-2 infection-a review of immune changes in patients with viral pneumonia. Emerg Microbes Infect 2020; 9: 727-732.

11 Tang N, Bai H, Chen X, et al. Anticoagulant treatment is associated with decreased mortality in severe coronavirus disease 2019 patients with coagulopathy. J Thromb Haemost 2020; 18: 1094-1099.

12 Bunce PE, High SM, Nadjafi M, et al. Pandemic H1N1 influenza infection and vascular thrombosis. Clin Infect Dis 2011; 52: e14-e17.

Copyright (CERS 2020

This version is distributed under the terms of the Creative Commons Attribution Non-Commercial Licence 4.0. 